\title{
INFLAMMATORY RESPONSE IN SOLID ORGAN AND TISSUE RECIPIENT WITH COVID-19
}

\author{
Ana Teresa Sobreira Lima Verde, Ana Vitória Magalhães Chaves, Fernando Barroso Duarte, José \\ Huygens Parente Garcia e Paula Frassinetti Castelo Branco Camurça Fernandes
}

All authors from Hospital Universitário Walter Cantídio, Fortaleza, Ceará

Correspondence to: anateresa.sobreira@gmail.com

\begin{abstract}
Introduction: The COVID-19 infection is caused by the new severe acute respiratory syndrome coronavirus 2 (SARS-CoV-2)-infection, which was first reported in Hubei, Wuhan province, China, in December 2019. There is a concern that immunocompromised patients are at greater risk of morbidity and mortality due to COVID-19 infection, although there is limited data on these patients. Here, we present an evolution of a series of cases of patients with COVID-19 in our service.
\end{abstract}

Patients and methods: This is a retrospective cohort study conducted at the Hospital Universitário Walter Cantídio in Fortaleza-CE, Brazil. All patients hospitalized due to COVID-19 were screened for a history of organ or tissue transplantation, with a total number of 77 patients. Only patients confirmed for COVID-19 were included in the study. The inflammatory response and initial laboratory results, as well as the CALL score, were compared to a cohort of patients with COVID-19 not transplanted at the same time in our clinical ward or intensive care unit (ICU). The clinical course and clinical findings recorded during treatment were extracted from the electronic medical record. A bilateral $\mathrm{P}<0.05$ (5\%) was considered significant. Results: The total number of hospitalizations until July 24, 2020 for confirmed cases of COVID-19 was 77 patients. Of the total, 33 (42\%) patients needed ICU. Most patients were male (61\%). The median age was 62 [95\% Cl: 54-63] years, 31 (37\%) had a previous diagnosis of hypertension, 24 (28\%) of type 2 diabetes mellitus (DM-2). The total lethality of our service was $22 \%$. The CALL score of patients admitted to the clinical ward and in the ICU was analyzed, with a higher average observed in the patients admitted in ICU, the average was 9.34 in the patients admitted in the clinical ward and 10.9 in the patients who required ICU. $(p=0.003)$. The effect of neutrophil/lymphocyte ratio(NLR) at admission on the need of ICU care was analyzed by ROC curve and AUC and was found to be significant (AUC: 0.708, $\mathrm{p}=0.002,95 \% \mathrm{Cl}=0.593$ to 0.823 ). The number of transplant recipients in our service was 17 patients. The mean age was 56 years and the median was 55 years [95\% Cl: 45-65 years]. Of this subgroup, 6 patients (35\%) required ICU, with no statistical difference when compared to non- transplanted patients $(p=0.443)$, and only 3 evolved to death $(17 \%)$, also without statistical difference when compared to the subgroup of non-transplanted patients $(p=0.484)$. When compared to the sample of non-transplanted patients, lower values were found of the White Blood cells count, neutrophils and ferritin. However, despite lower values of fibrinogen, D-dimer, C-reactive protein (CRP) and lactate dehydrogenase (LDH), there was no statistical difference.

Conclusion: It is a new disease, with few data, mainly in the studied population. Our sample was a reduced sample, however it was surprising to see a lower inflammatory tendency, although without statistical significance and with mortality similar to the general population. In addition, it is worth emphasizing the importance shown on the neutrophil / lymphocyte ratio of admission demonstrated by the ROC curve in patients who evolve in need of an ICU care.

Keywords: Inflamatory response, Transplant, Kidney, Liver, Covid-19 


\section{INTRODUCTION}

The COVID-19 infection is caused by the new severe acute respiratory syndrome coronavirus 2 (SARSCoV-2)-infection, which was first reported in Hubei, Wuhan province, China, in December 20191. With rapid spread, on March,11 2020, the World Health Organization declared pandemic situation. In Brazil, the overall disease mortality rate is $3.6 \% 2$. Mortality appears to be age-dependent, with higher rates among older adults (age 50-59: 1.3\%, 60-69: 3.6\%, 70-79: $8 \%, 80+: 14.8 \%) 3$. Mortality among the transplanted population appears to be higher in lung transplant recipients and lower in liver and heart transplant populations4. There is a concern that immunocompromised patients are at greater risk of morbidity and mortality due to COVID-19 infection, although there is limited data on these patients. Here, we present an evolution of a series of cases of patients with COVID-19 in our service.

\section{PATIENTS AND METHODS}

This is a retrospective cohort study conducted at the Hospital Universitário Walter Cantídio in Fortaleza-CE, Brazil. All patients hospitalized due to COVID-19 were screened for a history of organ or tissue transplantation, with a total number of 77 patients. Only patients confirmed for COVID-19 were included in the study.

Between the 77 patients, 17 were solid organ or tissue recipient patients, with 14 solid organ transplanted patients (8 patients with kidney transplant, 6 patients with liver transplant) and 3 patients were bone marrow recipients. The inflammatory response and initial laboratory results, as well as the CALL score (score that evaluates prognosis based on the variables comorbidity, age, lymphocyte levels and lactate dehydrogenase) 5, were compared to a cohort of patients with COVID-19 not transplanted at the same time in our clinical ward or intensive care unit (ICU). The clinical course and clinical findings recorded during treatment were extracted from the electronic medical record. Categorical variables were frequency and percentage rates and continuous variables by median and interquartile range (IQR). Significance was tested using the Kruskal - Wallis test or Fisher's exact test. The performance of neutrophil/ lymphocyte ratio(NLR). was assessed using receiver operating characteristic (ROC) curve analysis and by calculating the area under the curve (AUC) of the ROC curves. A bilateral P $<0.05(5 \%)$ was considered significant.

\section{RESULTS}

\section{General}

The total number of hospitalizations until July 24, 2020 for confirmed cases of COVID-19 was 77 patients. Of the total, 33 (42\%) patients needed ICU. Most patients were male (61\%). The median age was 62 [95\% Cl: 54-63] years, 31 (37\%) had a previous diagnosis of hypertension, 24 (28\%) of type 2 diabetes mellitus (DM-2). The blood count and inflammatory markers of admission are assigned to table 1. The total lethality of our service was $22 \%$. The CALL score of patients admitted to the clinical ward and in the ICU was analyzed, with a higher average observed in the patients admitted in ICU, the average was 9.34 in the patients admitted in the clinical ward and 10.9 in the patients who required ICU. $(p=0.003)$ (figure 1). The effect of neutrophil/lymphocyte ratio (NLR) at admission on the need of ICU care was analyzed by ROC curve and AUC and was found to be significant (AUC: $0.708, p=0.002,95 \% \mathrm{Cl}=0.593$ to 0.823 ) (figure 2).

\section{Transplanted patients}

The number of transplant recipients in our service was 17 patients. The mean age was 56 years and the median was 55 years [18-80]. Of this subgroup, 6 patients (35\%) required ICU, with no statistical difference when compared to non-transplanted patients $(p=0.443)$, and only 3 evolved to death $(17 \%)$, also without statistical difference when compared to the subgroup of non-transplanted patients ( $p=0.484)$. A descriptive analysis of the CALL score of the group of non-transplanted and transplanted patients is shown in figure 3 . The blood count and inflammatory markers of admission are available in table 2 . When compared to the sample of non-transplanted patients, lower values were found of the White Blood cells count, neutrophils and ferritin, however, despite lower values of fibrinogen, D-dimer, C-reactive protein (CRP) and lactate dehydrogenase (LDH), there was no statistical difference.

\section{Kidney transplant}

The number of kidney transplant recipients admitted to our service by COVID-19 confirmed was 8 patients. The mean age was 56 years ( $28-80$ years) and median 54 years. Of these, 3 required an ICU care and 2 died. Lethality in this subgroup was $25 \%$. Six patients were using Sirolimus and Tacrolimus at the time of admission, 1 patient was using cyclosporine associated with prednisone and 1 patient was using mycophenolate mofetil and tacrolimus. Only in pa- 
tients who evolved with severe acute respiratory failure, immunosuppressants were removed.

\section{Liver transplant}

The number of liver transplant recipients admitted to our service by COVID-19 confirmed was 6 patients. The mean age was 59 years ( $46-69$ years) and the median was 60 years. Of these, 2 needed an ICU bed and 1 died. Lethality in our service for this subgroup was $14.1 \%$. Patients remained on previous immunosuppressants, however, in two patients it was necessary to remove them after severe hemodynamic instability (severe acute respiratory failure requiring orotracheal intubation).

\section{Bone marrow transplant}

Only 3 allogeneic bone marrow recipients were admitted, only one of them required ICU. The mean age was 39 years (18-70 years) and the median was 31 years. In this subgroup, there was no death. Two patients were male. There was no statistical difference between the subgroup of transplant patients.

\section{DISCUSSION}

In the present study, a cohort of 77 laboratory-confirmed positive COVID-19 cases is reported. The median was 60 years with a greater preponderance of males $(61 \%)$ in consensus with other published cohorts. 6,7,8 The most common comorbidities were hypertension and diabetes, with a prevalence of $37 \%$ and $28 \%$, respectively. In the laboratory exams on admission, it was observed that higher values in patients who died of the WBC, number of neutrophils, of the NLR, fibrinogen and LDH with statistical significance. The values of hemoglobin, lymphocytes and monocytes, although reduced values were observed, had no statistical significance when comparing the groups of survivors and deaths, as well as in the inflammatory markers, C-reactive protein, $\mathrm{D}$-dimer and ferritin in which increased values were observed in both the groups but without statistical difference when compared.

The CALL score, created to predict progression risk in patients with COVID-19, analyzes 4 easily accessible variables: comorbidity, age, lymphocytes and LDH. The minimum score is 4 points and the maximum 13 points. In the original study, it was seen that a score greater than 6 points had a $50.7 \%$ chance of developing severe COVID-19 and below 6 points only a $1.5 \%$ chance of reaching the same outcome. 5 In our service, the mean of patients admitted to the clinical ward was 9.34 points and 10.9 points in patients who were admitted to the ICU, with statistical significance. However, when comparing the CALL score between living patients and deaths, there was no statistical significance between groups. Showing its benefit to assess prognosis and not outcome.

The overall hospital mortality of COVID-19 is approximately $15 \%$ to $20 \%$, but up to $40 \%$ among patients requiring admission to the ICU. However, mortality rates vary between cohorts, reflecting differences in test completeness and case identification, variable limits for hospitalization and differences in results. Hospital mortality ranges from less than $5 \%$ among patients under 40 years of age to $35 \%$ for patients aged 70 to 79 years and greater than $60 \%$ for patients aged between 80 and 89 years. 9 The total lethality of our service was $22 \%, 42 \%$ in patients admitted to the ICU. Regarding the age group at our service, there was no death below 40 years, $45 \%$ mortality between 70 and 79 years and $50 \%$ between 80 and 89 years.

Like other respiratory viral diseases, such as influenza, it is believed that deep lymphopenia can occur in individuals with COVID-19 when SARS-CoV-2 infects and destroys T lymphocyte cells. In addition, the viral inflammatory response, which it consists of the innate and adaptive immune response (comprising humoral and cell-mediated immunity), impairs lymphopoiesis and increases lymphocyte apoptosis.10 Therefore, Nalbatant et al showed the relationship between NLR as an independent predictor for the diagnose of COVID-19. 11 We analyzed NLR in patients admitted to the ICU, showing an independent variable, with an area under the curve of 0.708 ( $p=0.002,95 \% \mathrm{Cl}=0.593$ to 0.823 ). When comparing the group of living patients and deaths, RNL was higher in the group of deaths, with an average of 8.2 in living patients and 12.9 in patients who died, $p=$ 0.03 . This shows that this relationship can be a valuable low-cost tool to assess outcome in patients with COVID-19. However, more robust studies are needed to reach an adequate conclusion.

The immune response of organ receptors, particularly the immune response of T cells, is suppressed due to the long-term use of immunosuppressive agents. In recipients with COVID - 19 who develop extensive pneumonia, which may require intubation, our current therapeutic approach includes stopping immunosuppressive therapy (using steroids as the only anti-rejection drugs) to help promote the specific antiviral immune response. In our group of transplant patients, the median age was 55 years. In our population, $35 \%$ of this group needed ICU care, versus 
$42 \%$ of the group of non-transplant patients, however, with no statistical difference between them $(p=0.581)$. Higher rates of inflammatory markers were noted in the non-transplanted population, but there was no statistical difference, except for ferritin, which had a statistical difference between groups with higher values in the transplanted population. The lethality rate was $17 \%$ in the group of transplant patients admitted to our service versus $22 \%$ of the non-transplant population, with no statistical difference between the groups. There is a limited number of studies that analyze this population and with small samples size, as in our study.

\section{CONCLUSION}

It is a new disease, with few data, mainly in the studied population. Our sample was a reduced sample, however, it was surprising to see a lower inflammatory tendency, although without statistical significance and with mortality like the general population. In addition, it is worth emphasizing the importance shown of the neutrophil / lymphocyte ratio of admission demonstrated by the ROC curve in patients who evolve in need of an ICU care. Further studies with a larger population are needed to reach an appropriate conclusion.

\section{REFERENCE}

1. ZhuN, ZhangD, WangW,LiX, YangB,SongJ et al. A novel coronavirus from patients with pneumonia in China, 2019. N Engl J Med 2020 v. 382, n.9, p. $727-733$

2. https://covid.saude.gov.br. acessado em $26 / 07 / 2020$ às $18 \mathrm{~h}$.

3. China CDC Weekly 2020, p.13-22.

4. https://tts.org/tid-about/tid-presidents-message/23-tid/tid-news/657-tid-update-and-guidance-on-2019-novel-coronavirus-2019-ncovfor-transplant-id-clinicians

5. Dong Ji, Dawei Zhang, Jing $\mathrm{Xu}$, Zhu Chen, Tieniu Yang, Peng Zhao, Guofeng Chen, Gregory Cheng, Yudong Wang, Jingfeng $\mathrm{Bi}$, Lin Tan, George Lau, Enqiang Qin,Prediction for Progression Risk in Patients With COVID-19 Pneumo- nia: The CALL Score, Clinical Infectious Diseases, ciaa414, https://doi.org/10.1093/cid/ciaa414

6. GuanWJ,NiZY,HuY,etal;ChinaMedical Treatment Expert Group for Covid-19. Clinical characteristics of coronavirus disease 2019 in China. N Engl $J$ Med. 2020;382(18):1708-1720. doi: 10.1056/ NEJMoa2002032

7. RichardsonS,HirschJS,NarasimhanM,etal; the Northwell COVID-19 Research Consortium. Presenting characteristics, comorbidities, and outcomes among 5700 patients hospitalized with COVID-19 in the New York City area. JAMA. 2020; 323(20):2052-2059. doi:10.1001/jama.2020.6775

8. DochertyAB,HarrisonEM,GreenCA, et al; ISARIC4C investigators. Features of 20133 UK patients in hospital with COVID-19 using the ISARIC WHO Clinical Characterisation Protocol: prospective observational cohort study. BMJ. 2020; 369:m1985. doi:10.1136/bmj.m1985

9. RichardsonS,HirschJS,NarasimhanM, et al; the Northwell COVID-19 Research Consortium. Presenting characteristics, comorbidities, and outcomes among 5700 patients hospitalized with COVID-19 in the New York City area. JAMA. 2020v. 323, n. 20, p. 2052-2059. doi:10.1001/ jama.2020.6775

10.Wiersinga WJ, Rhodes A, Cheng AC, Peacock SJ, Prescott HC. Pathophysiology, Transmission, Diagnosis, and Treatment of Coronavirus Disease 2019 (COVID-19): A Review [published online ahead of print, $2020 \mathrm{Jul} 10]$. JAMA. 2020;10.1001/ jama.2020.12839. doi:10.1001/jama.2020.12839

11.Nalbant Ahmet, Kaya Tezcan, Varim Ceyhun, Yaylaci Selçuk, Tamer Ali, Cinemre Hakan. Can the neutrophil/lymphocyte ratio (NLR) have a role in the diagnosis of coronavirus 2019 disease (COVID-19)?. Rev. Assoc. Med. Bras. [Internet]. 2020 June [cited 2020 Aug 16] ; 66( 6 ): 746-751. Available from: http://www.scielo.br/scielo.php?script=sci_arttext\&pid=S0104-42302020000600746\&lng=en. Epub July 20, 2020. https://doi. org/10.1590/1806-9282 
TABLE 1. - General ( $\mathrm{N}=77)$

\begin{tabular}{c|c|c|c}
\hline & ALIVES & DIED & P VALUE \\
\hline Hemoglobin* & 10.99 & 10.97 & 0.934 \\
\hline $\begin{array}{c}\text { White blood cell } \\
(4000-11000 / \mathrm{mm} 3)\end{array}$ & 8155 & 11873 & 0.045 \\
\hline $\begin{array}{c}\text { Neutrophil } \\
(1600-7500 / \mathrm{mm} 3)\end{array}$ & 6376 & 9918 & 0.033 \\
\hline $\begin{array}{c}\text { Lymphocyte } \\
(800-45500 / \mathrm{mm})\end{array}$ & 1128 & 925 & 0.386 \\
\hline NLR & 8.2 & 12.99 & 0.034 \\
\hline $\begin{array}{c}\text { Monocyte } \\
(800-1000 / \mathrm{mm})\end{array}$ & 485 & 623 & 0.324 \\
\hline $\begin{array}{c}\text { Platelet } \\
(150000-500000 / \mathrm{mm} 3)\end{array}$ & 199860 & 227030 & 0.485 \\
\hline $\begin{array}{c}\text { Fibrinogen } \\
(180-350 \mathrm{mg} / \mathrm{dL})\end{array}$ & 468 & 667 & 0.012 \\
\hline $\begin{array}{c}\text { D-dimer } \\
(<0.5)\end{array}$ & 3.45 & 1.93 & 0.52 \\
\hline $\begin{array}{c}\text { CRP } \\
(\leq 0.5 \mathrm{mg} / \mathrm{dL})\end{array}$ & 8.8 & 9.77 & 0.870 \\
\hline $\begin{array}{c}\text { Ferritin } \\
(28-365 \mathrm{ng} / \mathrm{mL})\end{array}$ & 592 & 1347 & 0.103 \\
\hline $\begin{array}{c}\mathrm{LDH} \\
(230-460 \mathrm{U} / \mathrm{L})\end{array}$ & 807 & 0.011 \\
\hline
\end{tabular}

NLR: Neutrophil/lymphocyte ratio, CRP: C-reactive protein (CRP) and LDH: lactate dehydrogenase * To male: $13.5-18 \mathrm{~g} / \mathrm{dL}$ and to female $12.0-16.0 \mathrm{~g} / \mathrm{dL}$.

GRAPHIC 1- CALL score analyses in clinical ward and ICU patients.

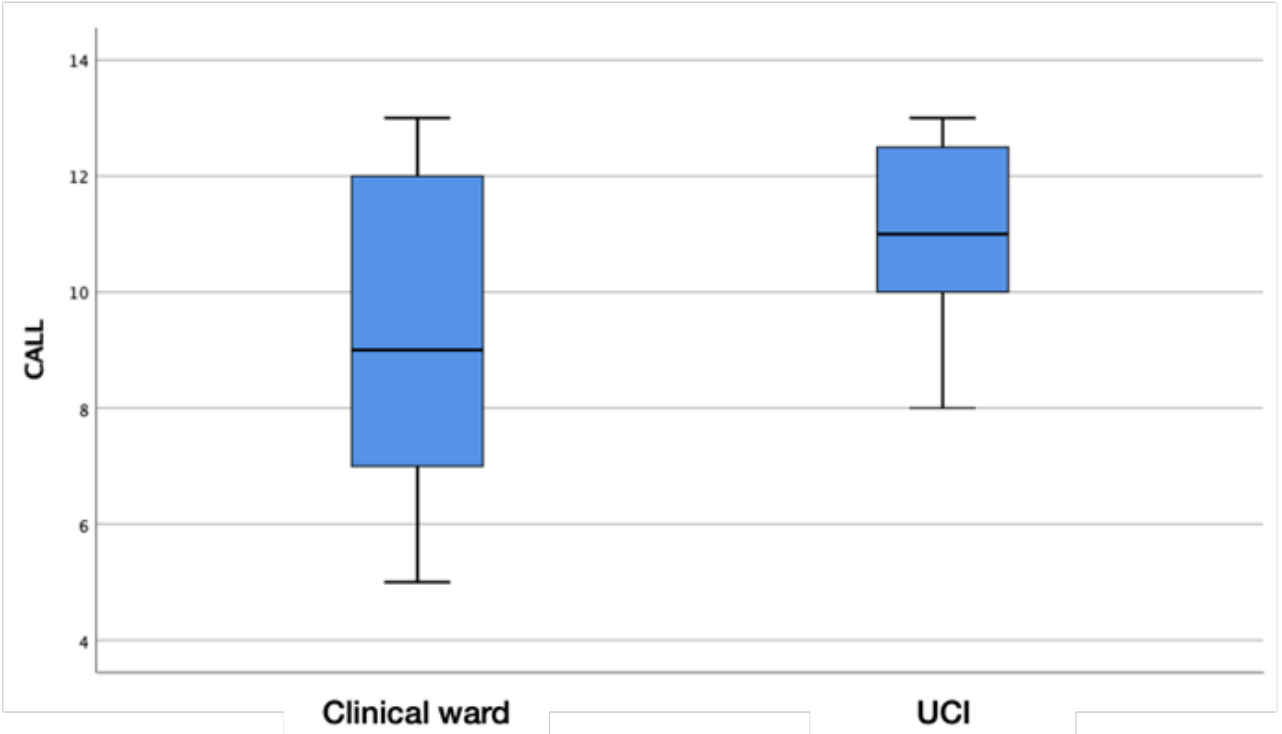


GRAPHIC 2 - ROC curve to neutrophil/lymphocyte ratio at admission on the need of ICU care

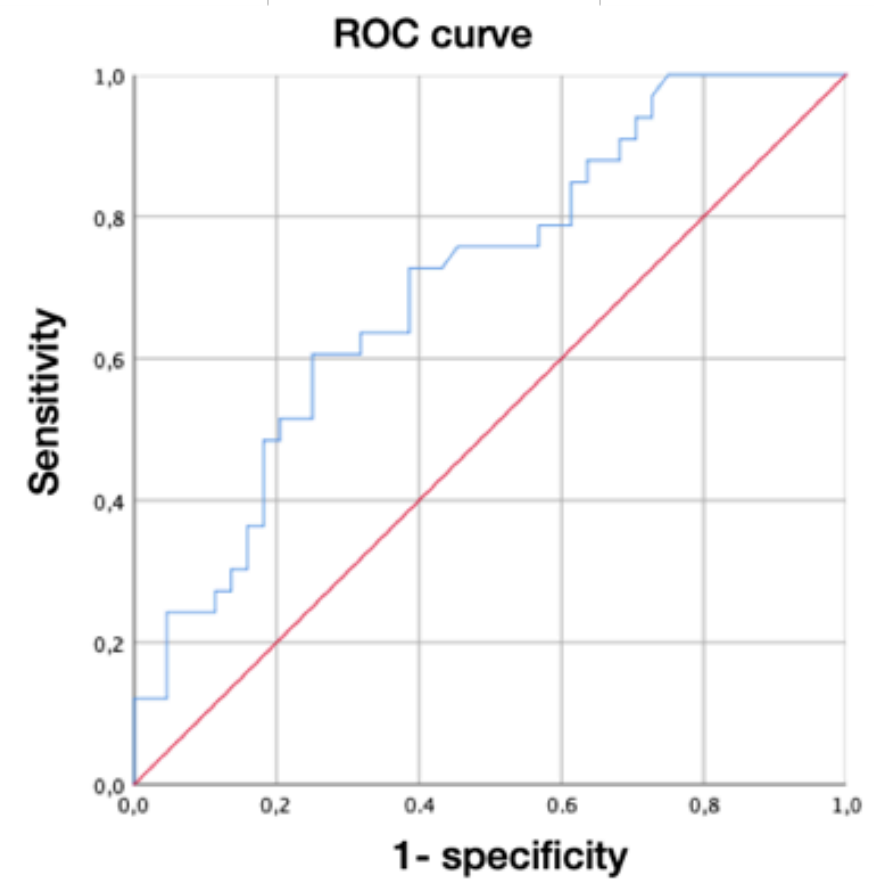

GRAPHIC 3 - Score CALL analyses in transplanted and non-transplanted patients

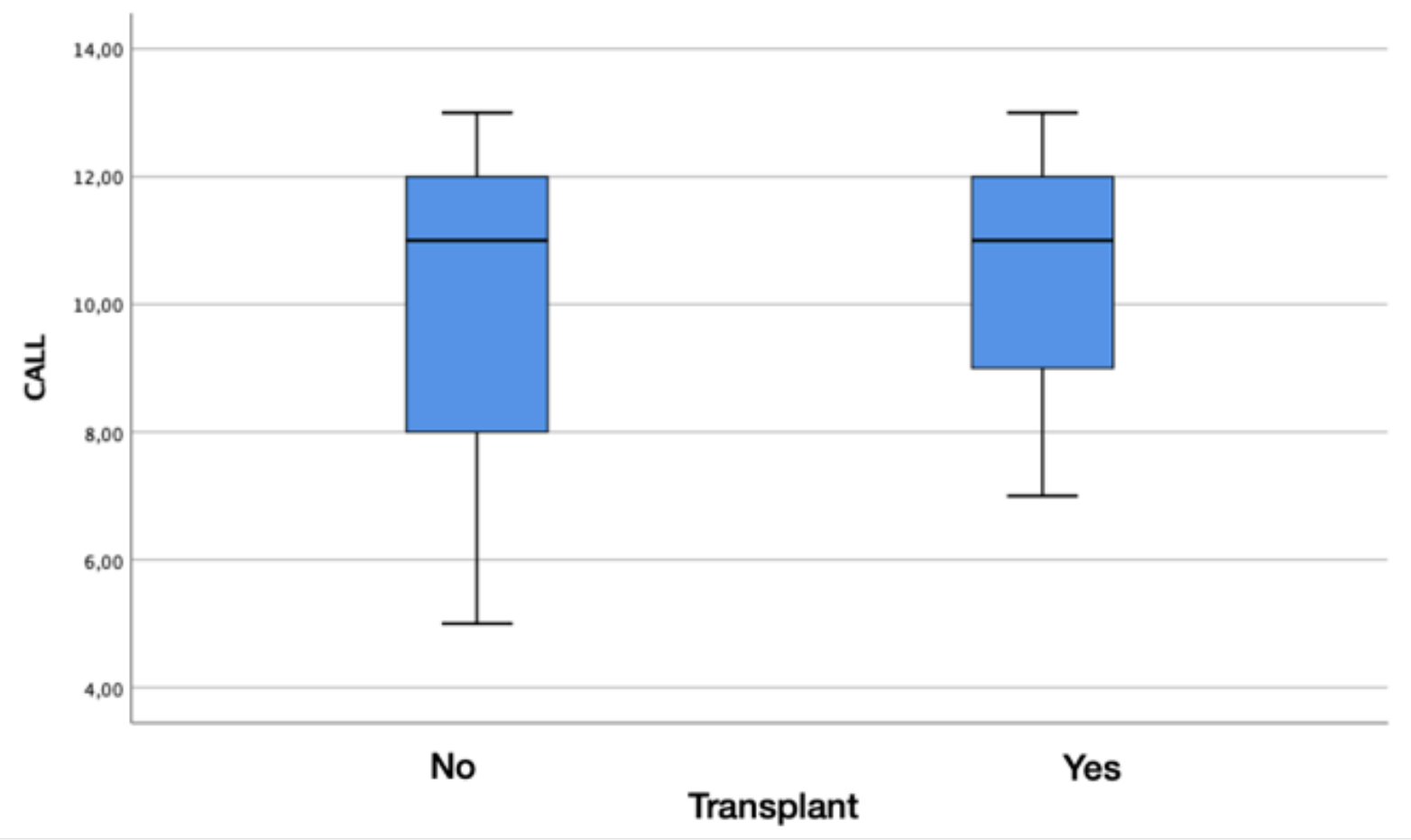


TABLE 2 - Admission blood count and inflammatory markers of transplanted and non-transplanted patients

\begin{tabular}{|c|c|c|c|}
\hline & TRANSPLANTED & NON-TRANSPLANTED & P VALUE \\
\hline Hemoglobin* & 9.76 & 10.82 & 0.107 \\
\hline $\begin{array}{l}\text { White blood cell } \\
(4000-11000 / \mathrm{mm} 3)\end{array}$ & 5280 & 10100 & 0.004 \\
\hline $\begin{array}{c}\text { Neutrophil } \\
(1600-7500 / \mathrm{mm} 3)\end{array}$ & 5280 & 8213 & 0.033 \\
\hline $\begin{array}{c}\text { Lymphocyte } \\
(800-45500 / \mathrm{mm} 3)\end{array}$ & 1076 & 1011 & 0.766 \\
\hline $\begin{array}{c}\text { Monocyte } \\
(800-1000 / \mathrm{mm} 3)\end{array}$ & 368 & 562 & 0.118 \\
\hline $\begin{array}{c}\text { Platelet } \\
(150000-500000 / \mathrm{mm} 3)\end{array}$ & 158284 & 213842 & 0.485 \\
\hline $\begin{array}{c}\text { Fibrinogen } \\
(180-350 \mathrm{mg} / \mathrm{dL})\end{array}$ & 468 & 667 & 0.012 \\
\hline $\begin{array}{l}\text { D-dimer } \\
(<0.5)\end{array}$ & 3.45 & 1.93 & 0.52 \\
\hline $\begin{array}{c}\text { CRP } \\
(\leq 0.5 \mathrm{mg} / \mathrm{dL})\end{array}$ & 8.8 & 9.77 & 0.870 \\
\hline $\begin{array}{c}\text { Ferritin } \\
(28-365 \mathrm{ng} / \mathrm{mL})\end{array}$ & 882 & 1347 & 0.103 \\
\hline $\begin{array}{c}\mathrm{LDH} \\
(230-460 \mathrm{U} / \mathrm{L})\end{array}$ & 592 & 807 & 0.011 \\
\hline
\end{tabular}

NLR: Neutrophil/lymphocyte ratio, CRP: C-reactive protein (CRP) and LDH: lactate dehydrogenase ${ }^{*}$ To male: $13.5-18 \mathrm{~g} / \mathrm{dL}$ and to female $12.0-16.0 \mathrm{~g} / \mathrm{dL}$. 